\title{
The ThreadBoard: Designing an E-Textile Rapid Prototyping Board
}

\author{
Chris Hill ${ }^{*}$ \\ Christian.N.Hill@colorado.edu \\ University of Colorado, Boulder \\ Boulder, $\mathrm{CO}$ \\ Ann Eisenberg \\ Ann.Eisenberg@colorado.edu \\ University of Colorado, Boulder \\ Boulder, $\mathrm{CO}$
}

\author{
Michael Schneider ${ }^{*}$ \\ Michael.J.Schneider@colorado.edu \\ University of Colorado, Boulder \\ Boulder, $\mathrm{CO}$ \\ Mark D. Gross \\ mdgross@colorado.edu \\ University of Colorado, Boulder \\ Boulder, $\mathrm{CO}$
}
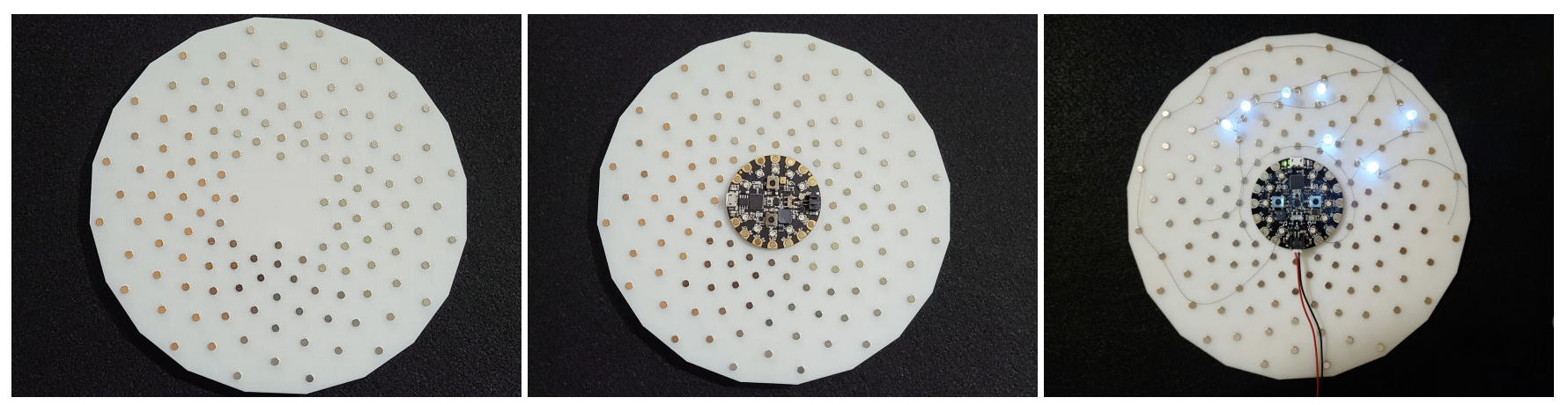

Figure 1: ThreadBoard with Adafruit Playground Classic

\begin{abstract}
E-textiles, which embed circuitry into textile fabrics, blend art and creative expression with engineering, making it a popular choice for STEAM classrooms [6, 12]. Currently, e-textile development relies on tools intended for traditional embedded systems, which utilize printed circuit boards and insulated wires. These tools do not translate well to e-textiles, which utilize fabric and uninsulated conductive thread. This mismatch of tools and materials can lead to an overly complicated development process for novices. In particular, rapid prototyping tools for traditional embedded systems are poorly matched for e-textile prototyping. This paper presents the ThreadBoard, a tool that supports rapid prototyping of e-textile circuits. With rapid prototyping, students can test circuit designs and identify circuitry errors prior to their sewn project. We present the design process used to iteratively create the ThreadBoard's layout, with the goal of improving its usability for e-textile creators.
\end{abstract}

*Both authors contributed equally to this research.

Permission to make digital or hard copies of all or part of this work for personal or classroom use is granted without fee provided that copies are not made or distributed for profit or commercial advantage and that copies bear this notice and the full citation on the first page. Copyrights for components of this work owned by others than ACM must be honored. Abstracting with credit is permitted. To copy otherwise, or republish, to post on servers or to redistribute to lists, requires prior specific permission and/or a fee. Request permissions from permissions@acm.org.

TEI '21, February 14-17, 2021, Salzburg, Austria

(c) 2021 Association for Computing Machinery.

ACM ISBN 978-1-4503-8213-7/21/02 . \$ \$15.00

https://doi.org/https://doi.org/10.1145/3430524.3440642

\section{CCS CONCEPTS}

- Human-centered computing $\rightarrow$ Interaction design.

\section{KEYWORDS}

E-textiles; rapid prototyping; e-textile tool

\section{ACM Reference Format:}

Chris Hill, Michael Schneider, Ann Eisenberg, and Mark D. Gross. 2021. The ThreadBoard: Designing an E-Textile Rapid Prototyping Board. In Fifteenth International Conference on Tangible, Embedded, and Embodied Interaction (TEI '21), February 14-17, 2021, Salzburg, Austria. ACM, New York, NY, USA, 7 pages. https://doi.org/https://doi.org/10.1145/3430524.3440642

\section{INTRODUCTION \& MOTIVATION}

E-textiles is a field of embedded computing that enables makers to design and construct computationally enriched textiles. An important technique that is present in traditional embedded computing development is missing from e-textile creation: rapid prototyping. Current e-textile tools for rapid prototyping either require makers to use custom components that will likely not be used in the end product, or they limit creativity by constraining makers to predesigned forms $[7,11,17]$. The need for rapid prototyping tools for e-textiles is highlighted by the difficulties associated with debugging threaded circuitry. Upon finding that their project does not perform as intended, e-textile makers face the disheartening task of disassembling their project to isolate and identify bugs. Once they find a bug and attempt a fix, the maker must then restitch portions of their project in order to test it. The typical novice strategy of 
trial-and-error is time intensive and frustrating with the regular unstitching and restitching of circuits. While some tools have been suggested to assist with locating errors in e-textile circuits, either during [3] or after the sewing process [14], we instead advocate for helping students iterate over their circuit's design before sewing, in a manner similar to the traditional solderless breadboard where the user can quickly disconnect and reconfigure hardware components and a microcontroller.

We have been working on a project to support young learners in acquiring skills in debugging, specifically in the domain of etextiles. As part of this project we aim to provide e-textile makers a platform that brings the benefits of the solderless breadboard to the world of e-textiles. Although our first group of intended users are middle-school (age 14) children, the ThreadBoard platform we have been developing is useful and appropriate for beginning e-textile makers of any age. Our ThreadBoard provides a layout of magnetic connectors that holds circuit components and conductive thread in place. This allows makers to rapidly prototype e-textile projects by quickly exploring and investigating different e-textile circuit designs, testing components, and finding and fixing bugs before committing to stitching the circuit into a fabric substrate.

Experimenters and developers of microcontroller projects are familiar with the solderless breadboard, a mainstay of prototyping traditional embedded systems. The breadboard layout consists of two power rails (Vcc and Ground, or + and -) in one direction, and perpendicular to the power rails, an array of terminal strips. The user connects components to configure a circuit by plugging short wires (jumpers) between sockets on the terminal strips and power rails. The breadboard makes it easy to reconfigure circuit topology, an enormous benefit for users as they prototype, test, and debug embedded system circuits. Once the circuit performs as intended, the user rebuilds the circuit, omitting the breadboard, either by laying out a solderable circuit board with the same topology or, if more than a few instances will be made, a printed circuit board. The solderless breadboard is essential for prototyping electronic circuits. However, for novices building e-textiles it has two serious drawbacks. First, novices find the breadboard initially confusing. They must map their intended circuit topology to the row-column layout of the breadboard, using terminal strips to connect components instead of connecting components directly to one another. The connections of most breadboards are hidden behind the plastic substrate, so novices find it difficult to grasp and remember the hidden circuit topology, e.g. two wires that are actually connected beneath the top plate of the breadboard do not appear to be connected. Second, whereas for typical electronic circuits prototyped on a breadboard the next step is a solderable or printed circuit board, for e-textiles the circuit is more likely to be stitched into a fabric substrate such as an article of clothing.

\section{BACKGROUND AND RELATED WORK ON E-TEXTILE PROTOTYPING}

Existing e-textile prototyping kits allow students to explore and create by either removing the need to sew or reducing the complexity of sewing required for prototyping an e-textile circuit. Some kits, like the Makershoe [7], Shirt Circuit [17], and TeeBoard [11], provide a pre-made wearable substrate (a shoe and t-shirts respectively) to which students connect components. This type of kit presents a tradeoff: students can quickly build functional prototypes but they must use the kit's custom components, which can only be placed at specific locations, limiting the variety of wearable projects the students can create.

Wearable Bits [5] offers more creative freedom. This kit consists of felt squares with pre-sewn components with which students can configure different types of wearables (eg. a hat or a shirt). This allows more flexibility in the e-textile projects the students can create; however students' circuits must fit within a grid and (similar to the TeeBoard) requires them to use alligator clips and button snaps to connect components. For greater creative freedom, kits like the LilyPad [1] provide components designed for wearable projects that a student can place anywhere they wish and connect with conductive thread. These components support prototyping by eliminating the need for sewing machines or complex techniques. Holes in the components enable students to sew their circuits together by hand. The key drawback is that, while the sewing is easier using (say) a LilyPad button vs a traditional button, students must still sew their prototypes together before they can be tested, which leads to a debugging cycle of stitching, unstitching, and restitching conductive thread circuits. To address this shortcoming, Craftec [4] modified the LilyPad and its components by attaching conductive fabric pads which can be sewn together to form a circuit. This does not remove the requirement of sewing, but instead limits it to just the point at which the fabric pads make contact.

E-textile kits can be arranged on a spectrum from highly structured to open system, or unstructured. On the highly structured end of the spectrum, by constraining choice the kits also help the maker avoid errors, however these kits restrict the creative freedom that, on the other end of the spectrum, open-ended kits afford. Kits such as the MakerShoe and TeeBoard fall on the highly structured end of this spectrum, Wearable Bits in the middle, and the LilyPad on the unstructured end. Beginning e-textile makers need a kit that falls somewhere between the easily assembled but restricting Wearable Bits and the unstructured but more challenging-to-assemble LilyPad. They need a kit that provides enough structure so they can quickly prototype their circuits but without restricting the variety of circuit designs they can create. The ThreadBoard addresses this need by providing a rapid prototyping board that uses components native to e-textiles and that allows free placement of conductive thread, sensors, actuators, and microcontrollers.

Instead of providing complete prototyping kits, some researchers have focused on adapting traditional embedded tools to work with e-textiles. Perner-Wilson created a "breadboard" pin cushion where the user pierces components through strips of conductive fabric that is adhered to a small cushion [13]. The pin cushion's "terminals" are configured similar to a traditional breadboard with power rails and terminal strips. This form enables testing individual components, like on a solderless breadboard, but does not support prototyping e-textile circuit designs.

To enable students to prototype their e-textile designs, Lo et al. [8] designed a simulation environment for drawing circuits called Ellustrate. Ellustrate provides functionality that blends the drawing capabilities of graphical program like Adobe Ilustrator with the circuit validation of a simulator, e.g. Autodesk's Tinkercad Circuit. This environment enables students to iterate over different designs 
for their circuit's pathways while preserving its aesthetic and artistic qualities. Once the design has been chosen, the student will then transfer the digital design to the physical median (conductive ink, copper tape, or conductive thread). Instead of utilizing a digital design space, the Threadboard provides a physical one where students may layout their conductive thread pathways and test with live hardware components. This lacks the automatic validation features of a simulator but does allow students to test their physical components before sewing the e-textile's circuit.

\section{DESIGN CONSIDERATIONS}

Our initial goals for ThreadBoard development were to:

- Provide an inviting interface for novices.

- Use materials and components that are native to e-textiles.

- Enable the creation of freeform circuitry.

- Support creative expression.

- Create a design that can be replicated with materials at home.

The first ThreadBoard (V1) was designed for a coin cell battery (Figure 2, left), which allows users to make basic circuit designs that support a single actuator. The design's stiff felt, to which the magnets were glued, could bend and fold around other objects. This allowed it to attach to flat or slightly curved metallic surfaces (e.g., a refrigerator or a water bottle) or to a metallic pin on the wearer's clothing. ThreadBoard V1 demonstrated that e-textile components-such as stainless steel conductive thread and LEDs-can be held in place by a magnet, enabling the construction of simple etextile circuits. However, ThreadBoard V1's size restricted creators to a small work area and did not allow for more complex projects, such as those that require a microcontroller.

The second ThreadBoard (V2), (Figure 3, left) changed the layout of the magnets to accommodate a $\mathrm{BBC}$ micro:bit. The magnets are arranged in columns aligned with the micro:bit's $\operatorname{GPIO}(0,1,2)$. power (3v), and ground (GND) pins. Also, as the LilyPad component connectors are not magnetic and therefore not attracted to either the ThreadBoard or conductive thread, we embedded magnets into the LilyPad components and taped them into place. Although only slightly larger than the initial ThreadBoard, the second version allowed for a microcontroller and e-textile electronics (conductive thread and LilyPad components), which enabled construction of more complex circuits, both in the variety of usable components and with the added ability to be controlled by software. However, due to the BBC micro:bit's pin layout, the ThreadBoard could not easily connect to its remaining pins, which lack alligator clip holes. This rules out circuits that use these pins to input sensor data or to control connected devices. Informal user testing revealed these limitations of size and pin access: a user could only connect two actuators - a LilyPad LED and vibration motor - to the "GND" and "V3" pin on the microcontroller (Figure 3, right). The small and compressed format made it difficult to employ the limited set of usable pins $(0,1,2)$ without risking the conductive thread crossing and causing a short circuit. This testing informed the next design iteration of the ThreadBoard to focus on creating a larger layout and designing for a microcontroller that is specifically designed for e-textile construction.
The current version (V3) of the ThreadBoard's layout is designed to support e-textile-specific microcontrollers like the LilyPad Arduino and Adafruit Circuit Playground (Figure 4, left). We also provided a larger area for making circuitry with the microcontroller placed at the center for aesthetic reasons, for example, and this suggested arranging the magnets in the floral disk pattern (Figure 4, right). This version of the ThreadBoard is sized at $192.9 \times 190.3 \times$ $3.0 \mathrm{~mm}$ with 128 holes for the magnets. The ThreadBoard is sized to fit several easily available $3 \mathrm{~d}$ printer beds such as the Creality Ender 3 and Prusa I3. The distance between the magnet holes is based on the distance between pins on e-textile components like the Adafruit Circuit Playground and LilyPad microcontrollers, as well as the LilyPad LED, vibration, and temperature components. Each hole is sized to fit a $4 \mathrm{~mm}$ disk magnet, magnets are adhered to the $3 \mathrm{~d}$ printed board using friction after being pressed into the hole. To present our methodology to the larger DIY community, we have created online documentation that provides steps on how to fabricate a V2 ${ }^{1}$ or $\mathrm{V}^{2}$ ThreadBoard.
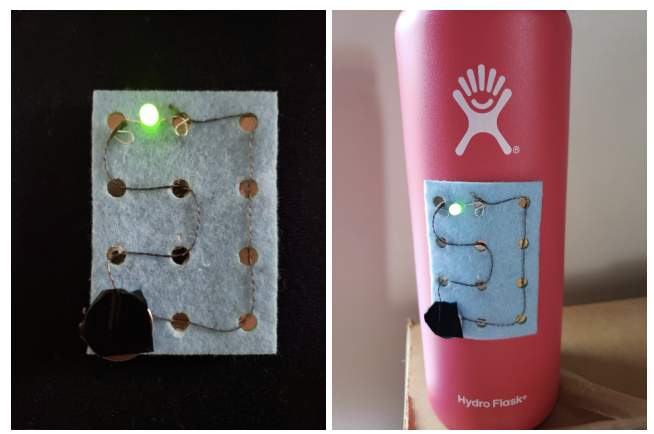

Figure 2: ThreadBoard V1 was designed for a coin cell battery; (left) a simple circuit; (right) mounted on a water bottle.
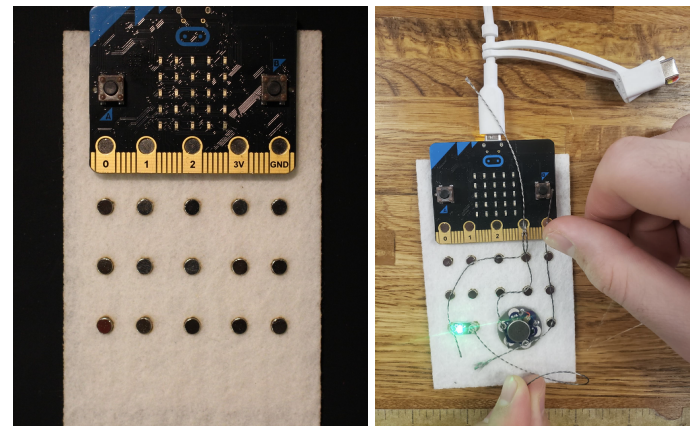

Figure 3: (left): Version 2 of the ThreadBoard designed for the BBC Micro:bit microcontroller. (right): E-textile circuitry designed by a user tester of the BBC Micro:bit version of the ThreadBoard

\footnotetext{
${ }^{1}$ https://www.instructables.com/The-ThreadBoard-Microbit-E-Textile-PrototypingBoa/

${ }^{2}$ https://makeprojects.com/project/the-threadboard-e-textile-prototyping-board
} 


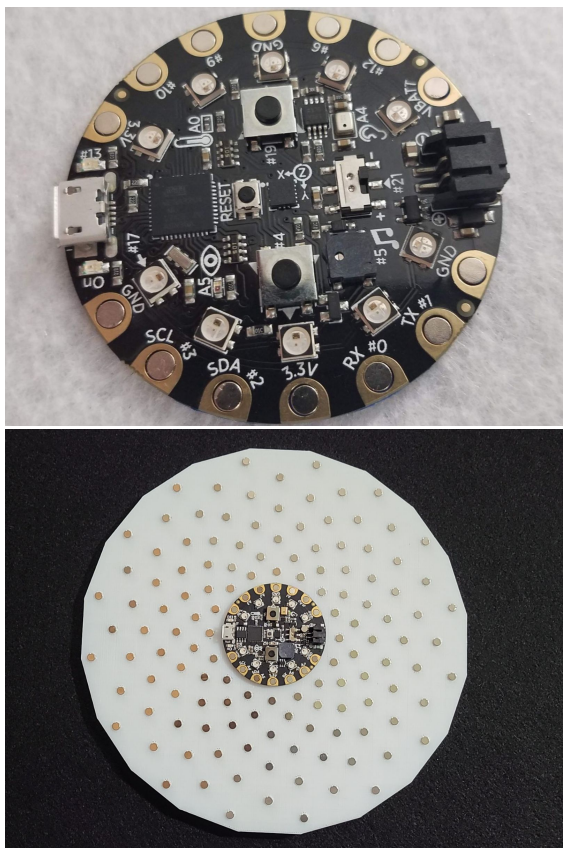

Figure 4: (left): A close-up of magnets laid into an Adafruit Circuit Playground. (right): A version of the ThreadBoard designed for the Adafruit Circuit Playground.

\section{EXPERT REVIEW}

\subsection{Expert Selection}

To understand how this version of the ThreadBoard might work in a classroom setting, we mailed it, along with a magnet enriched Adafruit Circuit Playground, conductive thread, and LEDs, to three experts in the e-textiles and education research community. We in terviewed the experts and observed them (remotely) as they crafted simple circuits with the ThreadBoard. Expert E1 (Leah Buechley) is a pioneer in the field of e-textiles with many years of experience inventing, teaching, and designing in this domain. She provided observations based on her experiences as a crafter and instructor. Experts E2 (Gayithri Jayathirtha) and E3 (Deborah Fields), also with years of experience, develop and teach e-textile curricula for middle school students. Their input provided insights into how the ThreadBoard might be used in the classroom and incorporated into existing STEAM curricula. Common themes across the three interviews and observations revealed potential strengths and weaknesses of the ThreadBoard for rapid prototyping.

\subsection{ThreadBoard as a Rapid Prototyping Tool}

A common concern was the need for rapid prototyping without losing an "authentic e-textile experience". E1 noted that the ThreadBoard "preserves some of the qualities of working with textiles while still letting you make a circuit quickly". E1 explained that students use alligator clips for testing components before sewing a circuit together (Figure 5). Although this allows for quick connections, it doesn't provide the desired "e-textile experience" because unlike conductive thread, the alligator clips are insulated and can be difficult to incorporate into larger circuits (e.g., providing a shared ground or power connection for several components). In contrast, the ThreadBoard allows students to prototype with conductive thread and e-textile components which E1 explains "introduces [them] to all the bugs [they] will encounter later. It helps [them] prepare for debugging in a way that existing prototyping tools do not. An anticipatory debugging tool."

\subsection{ThreadBoard as a Debugging Tool}

While the ThreadBoard is considered a rapid prototyping tool, experts E2 and E3 also noted the ThreadBoard's potential for improving the debugging experience. Expert E2 liked how the ThreadBoard could assist with "isolating code from circuitry-related concerns" which would help students to "get their code right before sewing". Experts E2 and E3 noted that their students first draw designs on paper, discuss them with an instructor, and then advance to sewing their circuits onto fabric. Student designs often exhibited "spatial errors", in which the orientation of a component or placement of a conductive thread pathway caused it to cross another, resulting in a short. These "spatial errors" often had simple fixes, such as repositioning a conductive thread pathway or reorienting a component, but students found the task of changing their already sewn circuits frustrating and time consuming. With the ThreadBoard, students could encounter these errors early and quickly alter their circuit's design (e.g. pick up and rotate the microcontroller or lay the conductive thread along a new path).

\subsection{ThreadBoard as a Creative Tool}

In addition to testing their circuit designs, the ThreadBoard also enables students to be creative and try different layouts of their circuit components, such as the Big Dipper scenario in Figure 5. Although all of our experts agreed that the ThreadBoard allows for artistic expression, expert E3 identified an unconscious bias of the ThreadBoard's circular design. E3 explained that because the Adafruit Circuit Playground is circular in form, students in e-textile workshops often created radial circuits. Likewise, the ThreadBoard's circular shape may increase students' proclivity towards radial circuit layouts. As tools used for creating strongly influence the thoughts and actions of the creators [15], E3's comments point to a potential need for a variety of ThreadBoard layouts. By providing different layouts to choose from, the ThreadBoard could allow for a greater variety in circuitry designs.

\section{FEATURES OF A MAGNET-BASED DESIGN}

\subsection{Embedded Magnets}

The ThreadBoard's form has changed with each design iteration, but magnets have remained a constant feature. Magnets provide a connector that makers can immediately understand and enjoy using [2]. Other connectors are employed in e-textile kits: alligator clips and button snaps are two of the most popular $[1,2,9,11$, 17]. Alligator clips require more dexterity and physical effort to open and keep open [2]. Likewise, sewn button snaps have similar physical constraints [2] and must be individually fabricated for each connection. We believe that magnets provide superior physical affordances for young makers. The ThreadBoard's magnets allow for components to be added, removed, and moved around with 


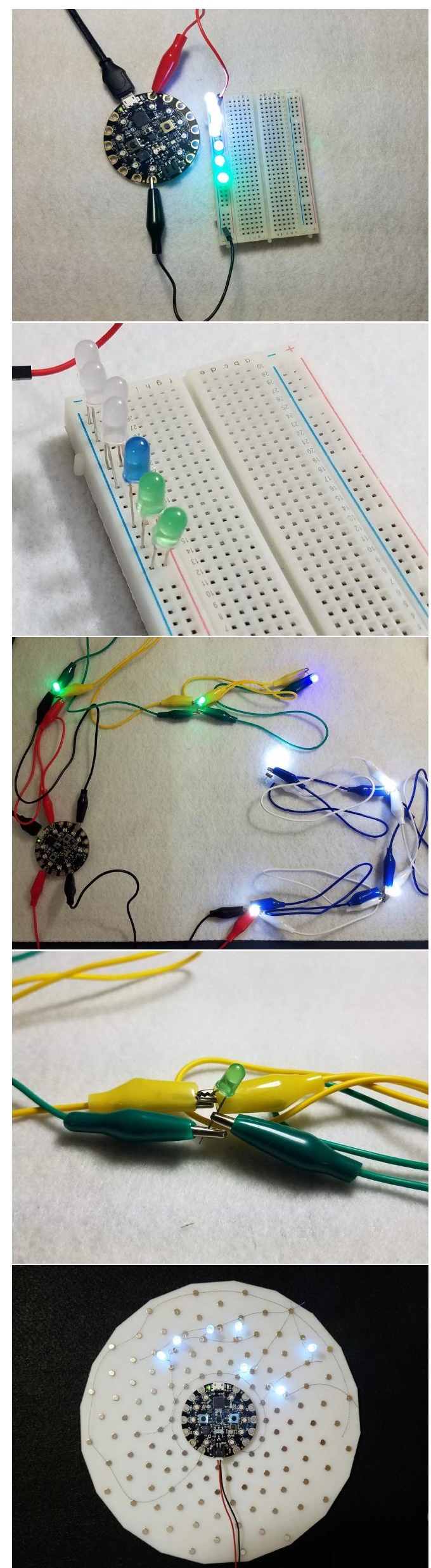

little effort compared to snaps and clips. However, magnets have a drawback: they lack the robust holding power of snaps, alligator clips, or even the traditional breadboard's sockets.

\subsection{Modifications of Existing E-Textile Components}

Sensors and actuators can be easily modified to interface with the ThreadBoard. Disk magnets placed in the component pins attract stainless steel thread to create connections and hold the component on the ThreadBoard. Other components like stainless steel thread, piezo buzzer, and standard LED are made of metals that are attracted to the embedded magnets. This allows prototyping e-textile circuits with the same components the sewn project will use, providing an authentic e-textile prototyping experience and simplifies the transition from prototype to end project.

\section{THE THREADBOARD AS PART OF THE E-TEXTILE ECOSYSTEM}

Both the traditional solderless breadboard and the ThreadBoard allow engineers to quickly prototype an electronic circuit by eliminating the need to solder wires or sew thread to connect components. However, while both tools reduce the difficulty of modifying prototypes, the ThreadBoard further simplifies circuit design by eliminating the need to understand the underlying (and hidden) conductive rails that connect the rows and columns of a breadboard. The ThreadBoard instead reveals the circuit as it will be embedded into the final project, hiding nothing from the maker.

Prototyping kits - in e-textiles and embedded systems at large - enable a designer to build a preliminary model of the behavior of the final product. However, a prototype need not have the same layout as the final product. Unlike prototyping using a conventional breadboard, which abstracts the layout of the final product, with the ThreadBoard, students can simultaneously investigate the behavior and layout of their e-textile design. For example, Figure 5 shows a set of LEDs arranged to form the "Big Dipper" constellation. With the ThreadBoard, a student can easily reconfigure the constellation's layout, whereas on a solderless breadboard a student can only arrange the LEDs into rows and columns, which limits them to testing only the LEDs' behavior and not exploring alternative arrangements. Alligator clips can be spaced in a similar fashion, but lack the flexible properties of conductive thread; they do not stay in place; and can require additional materials like conductive tape or fabric in order to share a common ground or power, a feature easily added to a ThreadBoard circuit (Figure 6).

Integrating behavior and layout provides two benefits. First, students can spot design flaws early in prototyping. As the ThreadBoard uses the non-insulated conductive thread ubiquitous in etextiles, students can inadvertently create shorts where threads touch or cross. If students find a short circuit in the prototyping phase, they can easily reconfigure the layout. The second benefit is that the ThreadBoard supports both "hard" and "soft" creators [16]. Hard creators are characterized by their preference "for abstract thinking and systematic planning", following a top-down planning approach. Soft creators, instead, prefer "a negotiable approach and concrete forms of reasoning”, allowing for bottom-up tinkering. For example, if a hard creator were to create the "Big Dipper" project 


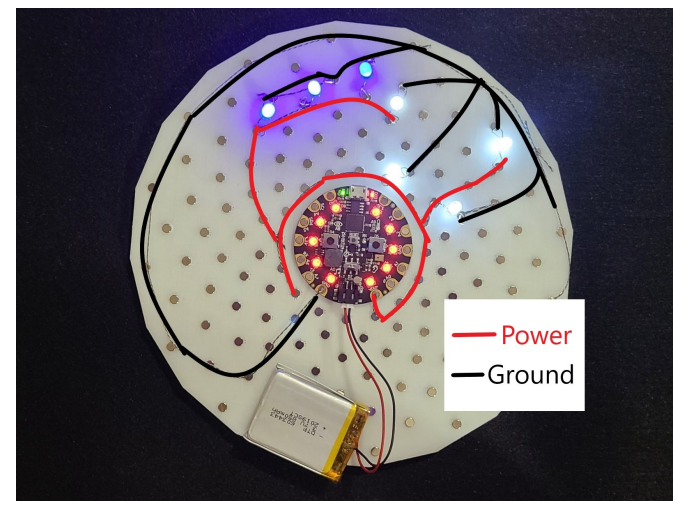

Figure 6: A diagram demonstrating shared ground and power on a ThreadBoard

(Figure 5), they would place and individually test components, verifying that the previous and current components match the expected behavior before placing and testing the next LED. A soft creator would place all components before laying down circuitry, verifying that the component placement represents their design, then begin testing the circuitry.

\section{LIMITATIONS AND FUTURE WORK}

\subsection{Time and Materials}

As mentioned above in our list of desiderata for the ThreadBoard, we aim for a platform that can easily be replicated at home or school. For this reason we intend to release the plans for the ThreadBoard as open-source. While the current version of the ThreadBoard works as proof-of-concept, ultimately we must address both the time and cost-intensive nature of building a ThreadBoard at home or for the classroom. The current design incorporates hundreds of tiny magnets which is costly and tedious to assemble. A possible solution is a more modular approach where the user inserts a magnet into the ThreadBoard only where they need a point of attachment for a component. Moreover, the ThreadBoard base is a 3D-printed disc, which takes four to six hours to fabricate with expensive filament. A laser-cut base would be cost-effective and relatively simple to make, but most hobbyists and classrooms do not have laser cutters. To address this we are experimenting with cutting thick/flexible foam or cardboard with a scrapbooking paper cutter (such as a Silhouette or Cricut). Due to the magnets' attraction to each other the ThreadBoard is currently designed to utilize a rigid substrate to hold the magnets in place. Because of this design limitation, some may perceive that the ThreadBoard is not an 'e-textile' prototyping tool due to its use of rigid plastics instead of flexible fabrics. A solution to this could be to provide or instruct users to place fabric over the ThreadBoard, and then begin placing their circuitry and components. This method will not affect the thread and components attraction to the board and may provide a more authentic textile experience.

\subsection{Geometric considerations}

Our early discussions with experts raised questions about the geometry of the ThreadBoard magnet layout and how those layouts affect circuit and project design. Additional studies will lend insight into the most useful magnet layout geometries for various groups of users, taking into account factors such as the user's level of expertise in electronics and e-textiles; types of microcontrollers and components used in the project; and the complexity of the project.

The current ThreadBoard is a rigid flat plate. We plan to modify this design into a three-dimensional prototyping device, which will more closely take the forms of many e-textile projects. We are beginning to work with a flexible three-dimensional mesh that can mold into a surface that mirrors the geometry of the target project, whether it is a stuffed animal, an article of clothing, or other fabric substrate. Figure 7 shows a 3d-printed piece of "chain mail" [10] with embedded magnets (left) and demonstrating the chain mail forming a bracelet (center) and a simple circuit (right). We are also experimenting with other materials to give the prototyping process a more textile-like aesthetic, as suggested by one of our reviewers.

\subsection{Computational extension with a PCB "hat"}

The addition of a microcontroller to the ThreadBoard requires magnets to be pressed into the alligator clip holes and either soldered in place or attached with adhesive tape. Unfortunately, neither option is ideal; magnets attached with tape may loosen over time (breaking the circuit) while soldering fixes the magnets as a permanent feature of the microcontroller, preventing its incorporation into the final project. Furthermore, some boards lack alligator clip connectors for each pin. The micro:bit only allows magnets to be applied to pins $0,1,2$, GND, and $3 \mathrm{~V}$, and the remaining (much narrower) pins can not interface with the ThreadBoard. As a solution, we plan to design a "hat" or "shield" that attaches to a microcontroller, enabling robust interfacing with the ThreadBoard. This will support a stronger connection while prototyping, while facilitating easy removal of magnets for assembly of the final project.

\section{CONCLUSION}

The ThreadBoard is a rapid prototyping platform for e-textiles that utilizes a system of magnets. Incorporating magnets provides a prototyping platform that is more appropriate for e-textiles than the solderless breadboard. Makers no longer need to work with single-use snap connectors or maneuver alligator clips onto small pins. Rather, they can now lay circuit traces with conductive thread. Moreover, without the opaque top layer substrate of a conventional breadboard, users can better visualize circuit connections, allowing them to more easily troubleshoot complex designs. Makers also do not need to fabricate or purchase special components for use with the ThreadBoard, as the magnets simplify modifying commercial electronic components. As a result, the ThreadBoard enables makers to build prototypes with components that can be directly transferred into a final product and have a more authentic e-textile experience.

\section{ACKNOWLEDGMENTS}

This work was supported by the Stem+C Award \#1742081 from the National Science Foundation. Any opinions, findings, and conclusions or recommendations expressed in this paper are those of the 


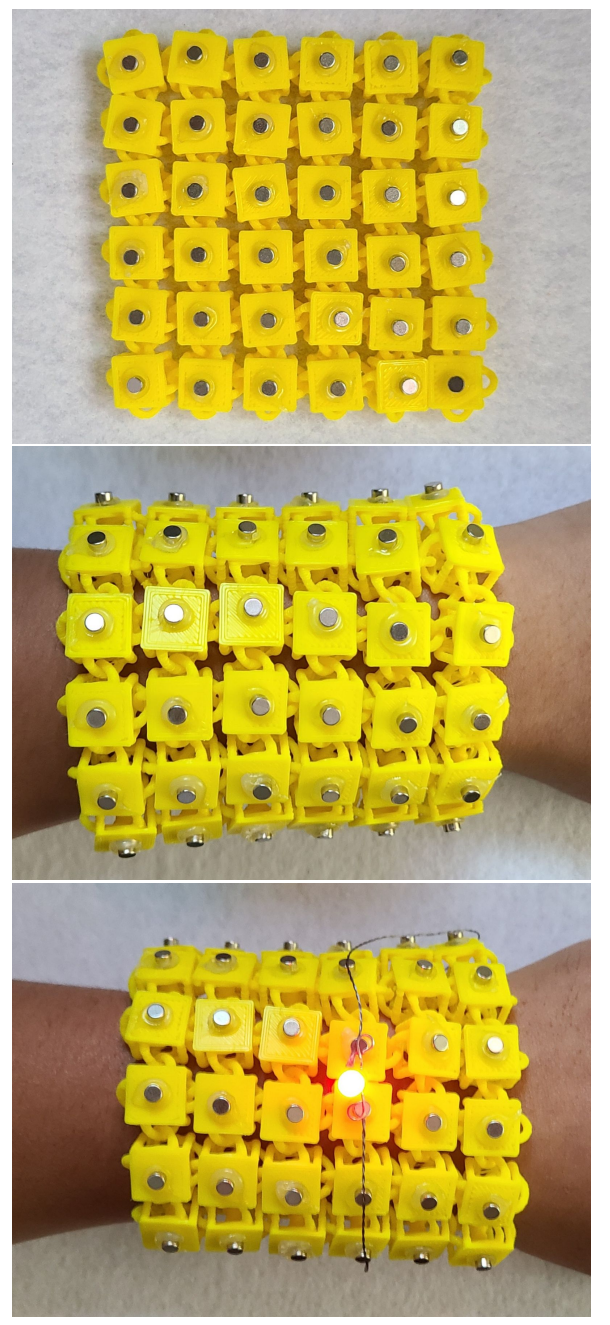

Figure 7: (Top) A 3D printed “chain mail” ThreadBoard. (Center) A bracelet formed from the chain mail ThreadBoard. (Bottom): An e-textile circuit prototyped on the chain mail ThreadBoard.

authors and do not necessarily reflect the views of the National Science Foundation or the University of Colorado, Boulder.

\section{REFERENCES}

[1] Leah Buechley and Michael Eisenberg. 2008. The LilyPad Arduino: Toward wearable engineering for everyone. IEEE Pervasive Computing 7, 2 (2008), 12-15. https://doi.org/10.1109/MPRV.2008.38

[2] Sharon Lynn Chu, Michael Saenz, and Francis Quek. 2016. Connectors in Maker Kits: Investigating Children's Motor Abilities in Making. In Proceedings of the The 15th International Conference on Interaction Design and Children. 452-462. https://doi.org/10.1145/2930674.2930714

[3] Chris Hill, Michael Schneider, and Mark Gross. 2020. A Wearable Meter That Actively Monitors the Continuity of E-Textile Circuits as They Are Sewn. (2020).

[4] Ben Jelen, Anne Freeman, Mina Narayanan, Kate M Sanders, James Clawson, and Katie A Siek. 2019. Craftec: Engaging older adults in making through a craftbased toolkit system. In Proceedings of the Thirteenth International Conference on Tangible, Embedded, and Embodied Interaction. 577-587.

[5] Lee Jones, Sara Nabil, Amanda McLeod, and Audrey Girouard. 2020. Wearable Bits: scaffolding creativity with a prototyping toolkit for wearable e-textiles. In Proceedings of the Fourteenth International Conference on Tangible, Embedded, and Embodied Interaction. 165-177. https://doi.org/10.1145/3374920.3374954

[6] Yasmin B Kafai, Deborah A Fields, Debora A Lui, Justice T Walker, Mia S Shaw, Gayithri Jayathirtha, Tomoko M Nakajima, Joanna Goode, and Michael T Giang. 2019. Stitching the Loop with Electronic Textiles: Promoting Equity in High School Students' Competencies and Perceptions of Computer Science. In Proceedings of the 50th ACM Technical Symposium on Computer Science Education. 1176-1182. https://doi.org/10.1145/3287324.3287426

[7] Majeed Kazemitabaar, Leyla Norooz, Mona Leigh Guha, and Jon E Froehlich. 2015. MakerShoe: towards a wearable e-textile construction kit to support creativity, playful making, and self-expression. In Proceedings of the 14th International Conference on Interaction Design and Children. 449-452. https://doi.org/10.1145/ 2771839.2771883

[8] Joanne Lo, Cesar Torres, Isabel Yang, Jasper O’Leary, Danny Kaufman, Wilmot Li, Mira Dontcheva, and Eric Paulos. 2016. Aesthetic electronics: Designing, sketching, and fabricating circuits through digital exploration. In Proceedings of the 29th Annual Symposium on User Interface Software and Technology. 665-676.

[9] Debora Lui, Emma Anderson, Yasmin B Kafai, and Gayithri Jayathirtha. 2017. Learning by fixing and designing problems: A reconstruction kit for debugging etextiles. In Proceedings of the 7th Annual Conference on Creativity and Fabrication in Education. 1-8. https://doi.org/10.1145/3141798.3141805

[10] Connor M. 2017. NASA Chainmail Fabric by ConnorMPrints. https://www. thingiverse.com/thing:2437081

[11] Grace Ngai, Stephen CF Chan, Joey CY Cheung, and Winnie WY Lau. 2009. The TeeBoard: an education-friendly construction platform for e-textiles and wearable computing. In Proceedings of the SIGCHI Conference on Human Factors in Computing Systems. 249-258. https://doi.org/10.1145/1518701.1518742

[12] Kylie Peppler. 2013. STEAM-powered computing education: Using e-textiles to integrate the arts and STEM. Computer 9 (2013), 38-43. https://doi.org/10.1109/ MC.2013.257

[13] Hannah Perner-Wilson. 2017. Breadbaord Pincushion. https://www.instructables. com/id/Breadbaord-Pincushion/

[14] Irene Posch and Geraldine Fitzpatrick. 2018. Integrating Textile Materials with Electronic Making: Creating New Tools and Practices. In Proceedings of the Twelfth International Conference on Tangible, Embedded, and Embodied Interaction. 158-165. https://doi.org/10.1145/3173225.3173255

[15] Mitchel Resnick, Brad Myers, Kumiyo Nakakoji, Ben Shneiderman, Randy Pausch, Ted Selker, and Mike Eisenberg. 2005. Design principles for tools to support creative thinking. (2005).

[16] Sherry Turkle and Seymour Papert. 1992. Epistemological pluralism and the revaluation of the concrete. Fournal of Mathematical Behavior 11, 1 (1992), 3-33.

[17] Clement Zheng and Manasvi Lalwani. 2017. Shirt Circuit: DIY Wearable Breadboard Circuits. https://www.instructables.com/id/Shirt-Circuit-DIY-WearableBreadboard-Circuits/ 